\title{
Reducing CD4 Monitoring in Children on Antiretroviral Therapy With Virologic Suppression
}

\author{
Mary-Ann Davies, MD, PhD, * Nathan Ford, PhD, † Helena Rabie, MD, MSc, + Geoffrey Fatti, MD, MPH, $\S$ \\ Kathryn Stinson, PhD, *ף Janet Giddy, MD, \| Frank Tanser, PhD, ** Karl-Günter Technau, MD, MSc, ††

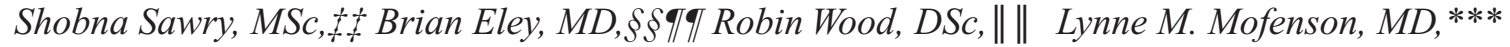 \\ Olivia Keiser, PhD, ††
}

Background: Ongoing CD4 monitoring in patients on antiretroviral therapy (ART) with viral suppression has been questioned. We evaluated the probability of CD4 decline in children with viral suppression and CD4 recovery after 1 year on ART.

Methods: We included children from 8 South African cohorts with routine HIV-RNA monitoring if (1) they were "responders" [HIV-RNA $<400$ copies $/ \mathrm{mL}$ and no severe immunosuppression after $\geq 1$ year on ART (time $0)$ ] and (2) $\geq 1$ HIV-RNA and CD4 measurement within 15 months of time 0 . We determined the probability of CD4 decline to World Health Organization-defined severe immunosuppression for 3 years after time 0 if viral suppression was maintained. Follow-up was censored at the earliest of the following dates: the day before first HIV-RNA measurement $>400$ copies/ $\mathrm{mL}$; day before a $>15$-month gap in testing and date of death, loss to followup, transfer out or database closure.

Results: Among 5984 children [median age at time 0: 5.8 years (interquartile range: 3.1-9.0)], 270 children experienced a single CD4 decline to severe immunosuppression within 3 years of time 0 with probability of $6.6 \%(95 \%$ CI: 5.8-7.4). A subsequent CD4 measurement within 15 months of the first low measurement was available for $63 \%$ of children with CD4 decline and $86 \%$ showed CD4 recovery. The probability of CD4 decline was lowest $(2.8 \%)$ in children aged 2 years or older with no or mild immunosuppression

\section{Accepted for publication March 22, 2015}

From the *Centre for Infectious Disease Epidemiology and Research, School of Public Health and Family Medicine, University of Cape Town, Cape Town, South Africa; †HIV/AIDS Department, World Health Organization, Geneva, Switzerland; †Tygerberg Academic Hospital, University of Stellenbosch, Stellenbosch, South Africa; §Kheth'Impilo, Cape Town, South Africa; - Médecins Sans Frontières, Khayelitsha, South Africa; $\|$ McCord Hospital, Durban, South Africa; **Hlabisa HIV Program, South Africa; ††Empilweni Services and Research Unit, Rahima Moosa Mother and Child Hospital, University of the Witwatersrand, Johannesburg, South Africa; Reproductive Health and HIV Institute, University of Witwatersrand, Johannesburg, South Africa; $\S \S R e d$ Cross War Memorial Children’s Hospital, University of Cape Town, Cape Town, South Africa; ब9Department of Paediatrics and Child Health, University of Cape Town, Cape Town, South Africa; || | Gugulethu HIV Program, Desmond Tutu HIV Centre, University of Cape Town, Cape Town, South Africa; ***Maternal and Pediatric Infectious Disease Branch, Eunice Kennedy Shriver National Institute of Child Health and Human Development (NICHD), Bethesda, Maryland; and $\dagger \dagger \dagger$ Institute of Social and Preventive Medicine, University of Bern, Bern, Switzerland.

Supported by the Eunice Kennedy Shriver National Institute of Child Health and Human Development and the National Institute of Allergy and Infectious Diseases (grant number 2U01AI069924-06; Egger, M.A.D.). The funders had no role in study design, data collection and analysis, decision to publish or preparation of the manuscript. H.R. has been paid by AbbVie for teaching activities unrelated to this manuscript. O.K. was supported by a PROSPER fellowship grant (nr 3233B 150934) from the Swiss National Science Foundation. The other authors have no conflicts of interest to disclose.

Address for correspondence: Mary-Ann Davies, MD, PhD, School of Public Health and Family Medicine, University of Cape Town Faculty of Health Sciences, Anzio Road, Observatory 7925, South Africa. E-mail: mary-ann. davies@uct.ac.za.

Supplemental digital content is available for this article. Direct URL citations appear in the printed text and are provided in the HTML and PDF versions of this article on the journal's website (www.pidj.com).

Copyright $(\subseteq 2015$ Wolters Kluwer Health, Inc. All rights reserved.

ISSN: 0891-3668/15/3412-1361

DOI: $10.1097 /$ INF.0000000000000912 and on ART for $<18$ months at time 0 . This group comprised $40 \%$ of children. Conclusions: This finding suggests that it may be safe to stop routine CD4 monitoring in children older than 2 years and rely on virologic monitoring alone.

Key Words: HIV-1, children, CD4, monitoring, viral load, sub-Saharan Africa, antiretroviral

(Pediatr Infect Dis J 2015;34:1361-1364)

$F$ or over 20 years, CD4 cell count measurements have been central to the management of patients with HIV to assess disease progression, guide decisions about antiretroviral therapy (ART) initiation and monitor treatment response. ${ }^{1,2}$ Although CD4 measurement continues to play an important role as a marker of disease progression and to identify the risk of opportunistic infections, the value of CD4 measurements for monitoring treatment response and diagnosing failure in settings where virologic monitoring is also available have come into question. ${ }^{3}$ Studies from the United States, ${ }^{4}$ Europe, ${ }^{5}$ Uganda $^{6}$ and South Africa ${ }^{7}$ have shown that, among adult patients who are stable on ART and virologically suppressed, CD4 decline is rare, and when it does occur, decline is usually transient, suggesting that repeated CD4 monitoring after viral suppression on ART is not necessary. Further CD4 measurement in a virally suppressed patient rarely contributes to clinical decision making. ${ }^{8}$ Studies among adult patients in the United States, ${ }^{9}$ the United Kingdom,,${ }^{10}$ Australia, Kenya $^{11}$ and South Africa ${ }^{12}$ have all estimated substantial programme cost savings if routine CD4 monitoring is reduced or stopped. Guidelines issued by the Southern African HIV Clinicians Society in 2013 recommend that for adult patients with routine viral load monitoring, there is no need to continue CD4 testing once CD4 is $>200$ cells/ $\mathrm{mm}^{3}$ and viral load is suppressed. CD4 testing is recommended if virologic or clinical failure occurs. ${ }^{13}$ In 2014, the World Health Organization (WHO) issued a technical document based on expert consultation, which concluded that if viral load is available routinely, CD4 monitoring could be reduced or stopped altogether in virologically suppressed patients. ${ }^{14}$ However, the absence of data for children was noted. We assessed CD4 changes in a cohort of virologically suppressed children on ART in South Africa.

\section{MATERIALS AND METHODS}

We conducted a retrospective analysis of data from 8 cohorts contributing to the International epidemiologic Databases to Evaluate AIDS Southern Africa (IeDEA-SA) collaboration (www.iedeasa.org). All cohorts have ethics approval from their respective local institutional review boards to contribute data to IeDEA-SA, and IeDEA-SA has been approved by the Human Research Ethics Committees of the Universities of Cape Town and Bern where the IeDEA-SA data centers are located.

Antiretroviral naïve children at sites with routine (at least annual) HIV-RNA and CD4 monitoring were included in the 


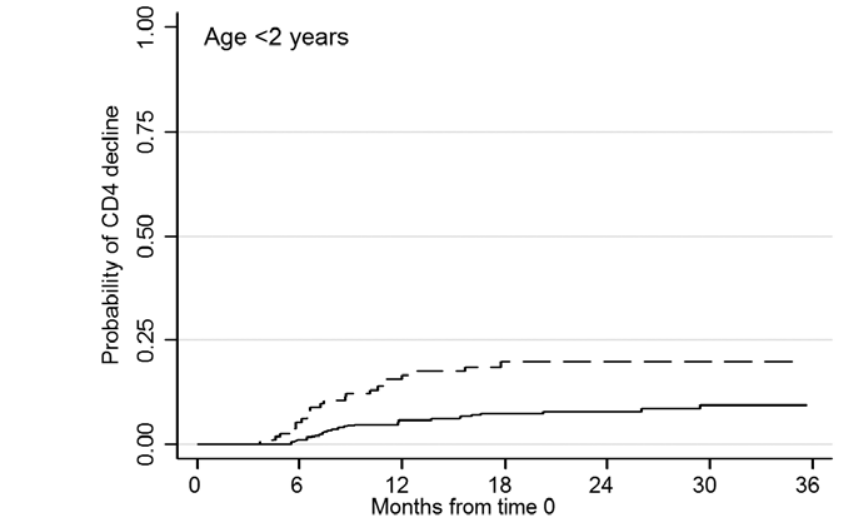

Number at risk

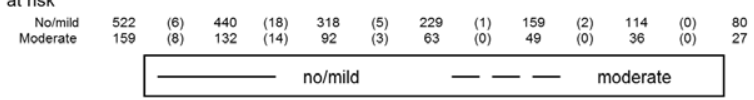

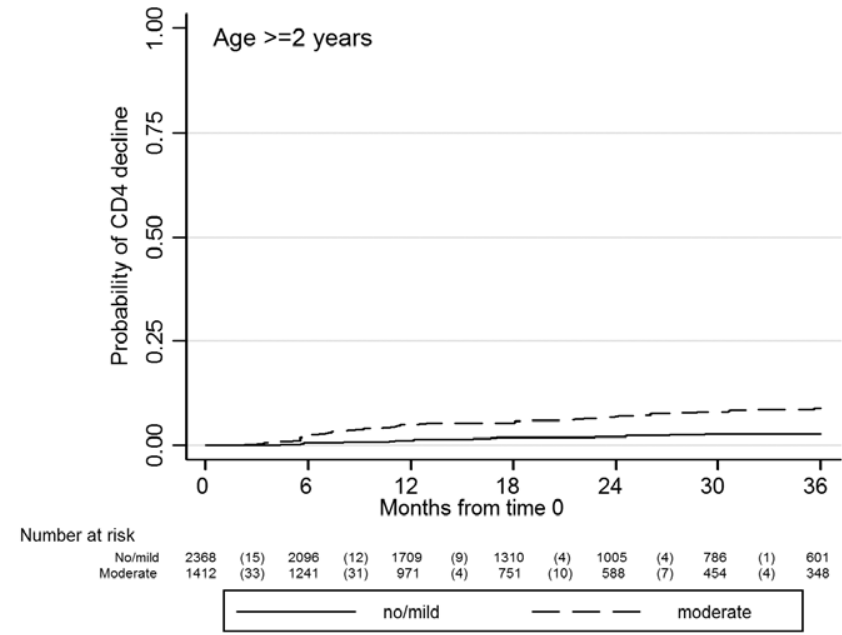

FIGURE 1. Probability of CD4 cell decline to severe immunosuppression for up to 3 years after time 0 according to degree of immunosuppression (no/mild vs. moderate) at time 0 . All children on ART for $<18$ months at time 0.

analysis if they had $\geq 12$ months follow-up on ART. Children entered the analysis on the first date that they met the criteria for an adequate response to treatment (defined as HIV-RNA $<400$ copies/mL and simultaneous CD4 with no severe immunosuppression after $\geq 9$ months on ART). The time when a child first met these criteria was designated time 0 . We evaluated all subsequent paired HIV-RNA and CD4 measures to determine the probability of a CD4 decline to severe immunosuppression after time 0 in children who remained virologically suppressed. Children who did not have at least 1 subsequent paired HIV-RNA and CD4 measurement within 15 months of time 0 were excluded. We used the WHO 2006 criteria to define severe immunosuppression: CD4 < 20\%/ 750 cells $/ \mathrm{mm}^{3}$ (age: $12-35$ months), CD4 $<15 \% / 350$ cells $/ \mathrm{mm}^{3}$ (age: $36-59$ months) and CD4 $<15 \% / 200$ cells $/ \mathrm{mm}^{3}$ (age: $\geq 5$ years). ${ }^{15}$ Data were censored the day before the first HIV-RNA measurement $>400$ copies $/ \mathrm{mL}$, the first $>15$-month gap in testing or last date of follow-up because of death, loss to follow-up (LTFU) or transfer out. LTFU was defined as no visit for 9 months before database closure.

Associations between characteristics at time 0 and CD4 decline were assessed using Cox-proportional hazards models. Multivariable models included variables considered a priori to affect the probability of CD4 decline, including age and duration on ART at time 0 and degree of immunosuppression both at time 0 and at ART initiation. Severe immunosuppression was defined as mentioned earlier. The definition of no/mild immunosuppression was based on the WHO criteria: CD4 $\geq 25 \%$ and 1000 cells $/ \mathrm{mm}^{3}$ in a child younger than 5 years or $\geq 20 \%$ and 500 cells $/ \mathrm{mm}^{3}$ in a child aged 5 years or older. ${ }^{15}$ Hazards proportionality was assessed by the analysis of scaled Schoenfeld residuals. All analyses were performed using Stata version 12.0 (College Station, TX).

\section{RESULTS}

Among 9503 children who had been on ART for $\geq 12$ months, 5984 met the inclusion criteria for this analysis. The reasons for exclusion are shown in Figure, Supplemental Digital Content 1, http://links.lww.com/INF/C263. The characteristics of included and excluded children are summarized in Table, Supplemental Digital Content 2, http://links.lww.com/INF/C264. Median (interquartile range) age at time 0 in children included in the analysis was 5.8 (3.19.0) years, and median (interquartile range) time on ART was 12.9 (11.3-18.0) months. First-line regimen information was recorded for
5696 (95.1\%) children, of whom 31.9\% initiated lopinavir-/ritonavirbased ART, whereas $61.6 \%$ and 3.5\% initiated efavirenz (EFV)- and nevirapine-based regimens, respectively. During the following 3 years, 270 children experienced a CD4 decline to severe immunosuppression, with a probability of $6.6 \%$ (95\% CI: 5.8-7.4). Most declines were not sustained: a subsequent CD4 measurement within 15 months of the first measurement was available for $169(63 \%)$ of 270 children with a CD4 decline; the majority $(\mathrm{n}=145,86 \%)$ no longer had severe immunosuppression at the next measurement. Only 3 of these children underwent a treatment change between the first and subsequent CD4 measurement: 1 child switched to second line [change of both nucleoside reverse transcriptase inhibitors and change from EFV to lopinavir/ritonavir (LPV/r)]; 1 child had a single-drug change from LPV/r to EFV and in the third child, ritonavir superboosting was added to the LPV/r-based regimen as tuberculosis treatment was started. Among the 270 children with CD4 decline, outcomes in the following year were as follows: $85.2 \%$ remained in care, $1.1 \%$ had died, $1.9 \%$ were lost to follow-up and $11.9 \%$ had been transferred to another site. In children with CD4 decline who remained in care but did not have a repeat CD4 measurement for inclusion in the analysis $(n=84)$, the main reason $(n=47)$ was that there was $<1$ year of follow-up from the date of low CD4 measurement to the date of database closure. Hence, there was insufficient time for a repeat CD4 as national guidelines recommended annual CD4 monitoring. Other reasons were that the next CD4 measurement was $>15$ months after the date of decline $(n=23)$ or that the child was not virologically suppressed at the next CD4 measurement $(n=8)$. Six children had no subsequent CD4 measurement despite $>365$ days of follow-up before database closure.

The 3-year probability of CD4 decline to severe immunosuppression was lowest $(2.8 \%$; 95\% CI: $2.1-3.8)$ in children with the following characteristics: $\geq 2$ years old at time 0 , met the criteria for an adequate response to ART within 18 months of starting treatment and good immunologic response (ie, had no/mild rather than severe immunosuppression at time 0 ). This subgroup comprised $40 \%(\mathrm{n}=2368)$ of all children included in the analysis (Fig. 1; see Table, Supplemental Digital Content 3, http://links.lww.com/INF/ $\mathrm{C} 265)$. The following characteristics at time 0 were associated with an increased risk of CD4 decline: age $<2$ years [adjusted hazard ratio (aHR): $2.80 ; 95 \% \mathrm{CI}: 1.95-4.01]$, moderate versus no/mild immunosuppression (aHR: 2.89; 95\% CI: 2.14-3.89) and taking longer than 18 months to respond adequately to ART (aHR: 1.51; 
95\% CI: 1.08-2.12). In addition, severe immunosuppression at ART initiation was associated with an increased risk of CD4 decline (aHR: 1.96 ; 95\% CI: $1.21-3.18)$. The groups with high ( $\geq 20 \%)$ risk of CD4 decline were those younger than 2 years at time 0 who either took $>18$ months to respond to ART or were still moderately immunosuppressed at time 0 . These comprised only $3.3 \%(n=195)$ of all children included in the study.

\section{DISCUSSION}

The value of continued CD4 monitoring for stable patients in settings where viral load is available has been questioned. The findings of our study carried out in a large pediatric population across several treatment sites in South Africa with both routine HIV-RNA and CD4 monitoring concur with reports among adult patients and suggest that the risk of CD4 decline in virologically suppressed children on ART is low, especially those aged $>2$ years who have attained no/mild immunosuppression. This finding indicates that it may be safe to reduce CD4 monitoring in virologically suppressed children aged $>2$ years on ART.

The risk of CD 4 decline was higher in those aged $<2$ years at entry into the study, especially if their immune responses were slower and less robust. As children had to have at least 12 months of follow-up on ART at study entry, these children would all have been 1-2 years of age at study entry, and so would have been followed up during the period when there is a biologic age-related rapid decline in CD4 count. ${ }^{16,17}$ This age-related decline may explain their increased risk of CD4 values falling to severe immunosuppression, especially if initial CD4 recovery was suboptimal. Further research needs to evaluate the clinical implications of CD4 decline at younger ages to determine whether there is a need for ongoing CD4 monitoring during this period of biologic rapid CD4 decline.

The current rationale for CD4 monitoring includes determining the need for ongoing co-trimoxazole prophylaxis; however, recent data have suggest that there is a benefit to continuing co-trimoxazole prophylaxis irrespective of CD4 count. A recent trial of co-trimoxazole prophylaxis in HIV-infected children on ART demonstrated substantial protection of prolonged co-trimoxazole against hospitalizations. ${ }^{18}$ Revised WHO 2014 guidelines recommended lifelong co-trimoxazole for HIV-infected children in settings where malaria and/or severe bacterial infections are highly prevalent. ${ }^{19}$

CD4 monitoring could also have a role in identifying patients at risk of opportunistic infections. In the context of ongoing virological suppression this need is likely to be small and the importance of this role depends on the availability and cost-effectiveness of interventions to diagnose and treat such illnesses. As our study lacked detailed data on opportunistic infections, we could not examine whether CD4 declines were associated with increased opportunistic infection risk, or whether there were any clinical interventions in response to low CD4 values. However, it is reassuring that most declines were transient without treatment changes in the majority of children and that mortality and LTFU in the year after a CD4 decline were low. However, our study was limited to South African sites with capacity for electronic data collection, and findings may not be generalizable to other regions of the world with children of different genetic background, ${ }^{16}$ different frequency of viral load measurement, antiretroviral regimens and opportunistic infection risk. Therefore, further research is encouraged to confirm these findings in other settings and over a longer duration.

The role of CD4 cell measurement in guiding treatment initiation for children may also be diminishing, given recent changes in guidelines for starting pediatric treatment. For programmatic reasons, WHO currently recommends that all adults, adolescents and children should be initiated on ART irrespective of CD4 cell count. ${ }^{20}$ Several countries are already providing or considering immediate ART to all children $<15$ years of age. ${ }^{21,22}$ However, although programmatically this may increase the current inadequate levels of pediatric treatment coverage, the current evidence regarding the individual clinical benefit of immediate ART initiation in children aged 5-14 years is limited, and a number of countries will likely continue to rely on CD4 to guide treatment initiation decisions for some years to come.

Thus, measurement of CD4 cell count will remain an important tool for assessing baseline health status, investigation for certain opportunistic infections and initiation of ART in older children. However, in settings where both CD4 and viral load testing are available and patients are stable on ART, this study suggests that ongoing routine monitoring of CD4 in children older than 2 years has limited additional value, and consideration should be given to reducing the frequency of $\mathrm{CD} 4$ monitoring or dropping its routine use altogether unless clinical deterioration or viral rebound occurs This could simplify programmes allowing for increased treatment access and for resources to be directed at ensuring that routine viral load measurement is undertaken satisfactorily.

\section{ACKNOWLEDGMENTS}

The authors thank the children whose data were used in this analysis, as well as their caregivers. They also thank all staff at participating sites for providing patient care and preparation of data contributed to the IeDEA-SA collaboration. They thank the IeDEA-SA Data Centre teams at the Universities of Cape Town and Bern.

IeDEA-SA Steering Group: Frank Tanser, Africa Centre for Health and Population Studies, University of Kwazulu-Natal, Somkhele, South Africa; Christopher Hoffmann, Aurum Institute for Health Research, Johannesburg, South Africa; Benjamin Chi, Centre for Infectious Disease Research in Zambia, Lusaka, Zambia; Denise Naniche, Centro de Investigação em Saúde de Manhiça, Manhiça, Mozambique; Robin Wood, Desmond Tutu HIV Centre (Gugulethu and Masiphumelele clinics), Cape Town, South Africa; Kathryn Stinson, Khayelitsha ART Programme and Médecins Sans Frontières, Cape Town, South Africa; Geoffrey Fatti, Kheth'Impilo Programme, South Africa; Sam Phiri, Lighthouse Trust Clinic, Lilongwe, Malawi; Janet Giddy, McCord Hospital, Durban, South Africa; Cleophas Chimbetete, Newlands Clinic, Harare, Zimbabwe; Kennedy Malista, Queen Elizabeth Hospital, Blantyre, Malawi; Brian Eley, Red Cross War Memorial Children's Hospital and Department of Paediatrics and Child Health, University of Cape Town, Cape Town, South Africa; Olatunbosun Faturiyele, SolidarMed SMART Programme, Lesotho; Michael Hobbins, SolidarMed SMART Programme, Pemba Region, Mozambique; Kamelia Kamenova, SolidarMed SMART Programme, Masvingo, Zimbabwe; Matthew Fox, Themba Lethu Clinic, Johannesburg, South Africa; Hans Prozesky, Tygerberg Academic Hospital, Stellenbosch, South Africa; Karl Technau, Empilweni Clinic, Rahima Moosa Mother and Child Hospital, Johannesburg, South Africa; Shobna Sawry, Harriet Shezi Children's Clinic, Chris Hani Baragwanath Hospital, Soweto, South Africa.

\section{REFERENCES}

1. Carpenter CC, Fischl MA, Hammer SM, et al. Antiretroviral therapy for HIV infection in 1996. Recommendations of an international panel. International AIDS Society-USA. JAMA. 1996;276:146-154.

2. Polk BF, Fox R, Brookmeyer R, et al. Predictors of the acquired immunodeficiency syndrome developing in a cohort of seropositive homosexual men. N Engl J Med. 1987;316:61-66.

3. Sax PE. Editorial commentary: can we break the habit of routine CD4 monitoring in HIV care? Clin Infect Dis. 2013;56:1344-1346.

4. Gale HB, Gitterman SR, Hoffman HJ, et al. Is frequent CD4+ T-lymphocyte count monitoring necessary for persons with counts $>=300$ cells $/ \mathrm{muL}$ and HIV-1 suppression? Clin Infect Dis. 2013;56:1340-1343. 
5. Whitlock GG, Ahmed N, Benn P, et al. Stop routine CD4 monitoring in HIVinfected patients with fully suppressed virus and CD4 $>=350$ cells $/ \mathrm{ml}$. Clin Infect Dis. 2013;57:327-328.

6. Reynolds SJ, Sempa JB, Kiragga AN, et al. Is CD4 monitoring needed among ugandan clients achieving a virologic and immunologic response to treatment? AIDS Patient Care STDS. 2014;28:575-578.

7. Ford N, Stinson K, Davies MA, et al. Is it safe to drop CD4+ monitoring among virologically suppressed patients: a cohort evaluation from Khayelitsha, South Africa. AIDS. 2014;28:2003-2005.

8. Chow EP, Read TR, Chen MY, et al. Routine CD4 cell count monitoring seldom contributes to clinical decision-making on antiretroviral therapy in virologically suppressed HIV-infected patients. HIV Med. 2015;16:196-200.

9. Hyle EP, Sax PE, Walensky RP. Potential savings by reduced CD4 monitoring in stable patients with HIV receiving antiretroviral therapy. JAMA Intern Med. 2013;173:1746-1748.

10. Duncan C, Schmid M, Schwab U, et al. Futility of CD4R monitoring in HIV-1 patients with CD4R cell count above 350 cells/ml on suppressive antiretroviral therapy. AIDS. 2014;28:2638-2639.

11. Kizito W, Ngoy B, Ritter H, et al. Is CD4 monitoring needed where there is routine viral load? A cohort analysis from Kibera, Kenya. In: MSF UK Scientific Day, May 2014. Available at: http://f1000.com/posters/browse/ summary/1095610. Accessed December 31, 2014.

12. Stevens WS, Ford N. Time to reduce CD4+ monitoring for the management of antiretroviral therapy in HIV-infected individuals. S Afr Med J. 2014;104:559-560.

13. Meintjes G, Boulle A, Conradie F, et al. Guidelines for antiretroviral therapy in adults by the Southern African HIV Clinicians Society. S Afr J HIV Med 2012;13:114-133.
14. WHO. March 2014 Supplement to the 2013 Consolidated Guidelines on the Use of Antiretroviral Drugs for Treating and Preventing HIV Infection. Recommendations for a Public Health Approach. 2014. Available at: www. who.int. Accessed April 1, 2014.

15. WHO. Antiretroviral Therapy of HIV Infection in Infants and Children: Towards Universal Access. 2006. Available at:http://www.who.int/hiv/pub/ paediatric/infants/en/index.html. Accessed September 2, 2007.

16. European Collaborative Study. Age-related standards for total lymphocyte, CD4+ and CD8+ T cell counts in children born in Europe. Pediatr Infect Dis J. 2005;24:595-600.

17. Lugada ES, Mermin J, Kaharuza F, et al. Population-based hematologic and immunologic reference values for a healthy Ugandan population. Clin Diagn Lab Immunol. 2004;11:29-34.

18. Bwakura-Dangarembizi M, Kendall L, Bakeera-Kitaka S, et al.; Antiretrovira Research for Watoto (ARROW) Trial Team. A randomized trial of prolonged cotrimoxazole in HIV-infected children in Africa. N Engl J Med. 2014;370:41-53.

19. WHO. Guidelines on Post-Exposure Prophylaxis for HIV and the Use of Cotrimoxazole Prophylaxis for HIV-Related Infections Among Adults, Adolescents, and Children. Recommendations for a Public Health Approach. 2014. Available at: http://www.who.int/hiv/pub/guidelines/arv2013/arvs2013upplement_dec2014/en/. Accessed March 15, 2015.

20. WHO. Guideline on when to start antiretroviral therapy and on pre-exposure prophylaxis for HIV. WHO; 2015.

21. Gonzales L. South Africa to move to earlier HIV treatment. Health-e Available at: http://www.health-e.org.za/2014/04/04/south-africa-moveearlier-hiv-treatment/. Accessed April 20, 2014.

22. WHO. Maximizing the treatment and prevention potential of antiretroviral drugs: early country experience towards implementing a treat-all policy. WHO; 2015. 